\title{
Big Bang Nucleosynthesis (BBN) and Non-Standard Physics
}

\author{
Tahani Makki ${ }^{1, \star}$ and Mounib El Eid ${ }^{1}$ \\ ${ }^{1}$ Department of Physics, American University of Beirut, Lebanon
}

\begin{abstract}
A brief overview on standard big bang nucleosynthesis (shortly, SBBN) is presented. First, we describe the outcome of the SBBN concerning the abundances of the light elements up to ${ }^{7} \mathrm{Li}$. A comparison with observations reveals a Lithium overproduction, which is not understood yet and is termed as "Cosmological Lithium Problem". Resolving that problem is not easy, since many aspects are involved which nuclear, astrophysical and even a non-standard scenario may be invoked. These items are described in some details owing to the limited available space.
\end{abstract}

\section{Introduction}

The standard big bang nucleosynthesis (SBBN) is well understood in the context of the standard model of particle physics. It predicts that the universe is composed of about $75 \%$ of Hydrogen and $25 \%$ of ${ }^{4} \mathrm{He}$ and small amounts of $\mathrm{D},{ }^{3} \mathrm{He},{ }^{7} \mathrm{Li}$ and ${ }^{6} \mathrm{Li}$ [4]. The most important nuclear reactions intervening in SBBN are given in Fig.1, while a comparison between SBBN calculations and observations is given in Table 1. It is clear that all abundances agree with observations except for Lithium which is higher by a factor of 3 than observations. This discrepancy constitute now one of the most intriguing problems in cosmology. In addition, Lithium was shown to have a constant behavior as function of metallicity, so called "Spite Plateau"[17]. Such a behavior is now in conflict with recent observations at very low metallicity which makes the Lithium problem more complicated. Solving the Lithium problem by taking into considerations accurate measurements of nuclear cross sections seems not possible [4]. In addition, the possibility of an astrophysical solution is also unlikely [13] because it will be constrained by the plateau at high metallicity.

\section{Non-Standard Big Bang Nucleosynthesis}

Including new particles/physics and changing fundamental constants were extensively studied to see the effect of these scenarios on SBBN or to constrain them by SBBN predictions. In addition, some non-standard scenarios were included for the aim to decrease the Lithium abundance. We mention a promising attempt by [7] where modifying the velocity distributions of nuclei during BBN have led to a decrease in Lithium to match observations.

In the following we give an example of non-standard scenario by adding extra dimension like dark component [1] and introducing a variable number of neutrinos and their chemical potential $(\beta)$. Increasing the number of neutrinos and/or adding $\beta$ will increase the standard radiation density $\rho_{\text {rad }}$

\footnotetext{
^e-mail: trm03@mail.aub.edu
} 

1. $\mathbf{n} \rightarrow{ }^{1} \mathrm{H}+\mathrm{e}^{-}+\overline{\mathbf{v}}$
7. ${ }^{3} \mathrm{H}+{ }^{4} \mathrm{He} \rightarrow{ }^{7} \mathrm{Li}+\boldsymbol{\gamma}$
2. ${ }^{1} \mathbf{H}+\mathbf{n} \rightarrow{ }^{2} \mathbf{H}+\gamma$
8. ${ }^{3} \mathbf{H e}+\mathbf{n} \rightarrow{ }^{3} \mathbf{H}+{ }^{\mathbf{1}} \mathbf{H}$
3. ${ }^{2} \mathrm{H}+{ }^{1} \mathrm{H} \rightarrow{ }^{3} \mathrm{He}+\gamma$
9. ${ }^{3} \mathrm{He}+{ }^{2} \mathrm{H} \rightarrow{ }^{4} \mathrm{He}+{ }^{1} \mathrm{H}$
4. ${ }^{2} \mathbf{H}+{ }^{2} \mathbf{H} \rightarrow{ }^{3} \mathrm{He}+\mathbf{n}$
10. ${ }^{3} \mathrm{He}+{ }^{4} \mathrm{He} \rightarrow{ }^{7} \mathrm{Be}+\gamma$
5. ${ }^{2} \mathbf{H}+{ }^{2} \mathbf{H} \rightarrow{ }^{3} \mathbf{H}+{ }^{1} \mathbf{H}$
11. ${ }^{7} \mathrm{Li}+{ }^{1} \mathrm{H} \rightarrow{ }^{4} \mathrm{He}+{ }^{4} \mathrm{He}$
6. ${ }^{2} \mathrm{H}+{ }^{3} \mathrm{H} \rightarrow{ }^{4} \mathrm{He}+\mathrm{n}$
12. ${ }^{7} \mathrm{Be}+\mathrm{n} \rightarrow{ }^{7} \mathrm{Li}+{ }^{1} \mathrm{H}$

Figure 1. The most important nuclear reactions for big bang nucleosynthesis.

Table 1. Comparison between SBBN predictions and observations.

\begin{tabular}{|c|c|c|}
\hline & Predictions & Observations \\
\hline$Y_{p}$ & 0.2457 & $0.2449 \pm 0.0040[2]$ \\
\hline $\mathrm{D} / \mathrm{H} \times 10^{5}$ & 2.6719 & $2.8_{-0.6}^{+0.8}[10]$ \\
\hline${ }^{3} \mathrm{He} / \mathrm{H} \times 10^{5}$ & 1.0193 & $1.1 \pm 0.2[3]$ \\
\hline${ }^{7} \mathrm{Li} / \mathrm{H} \times 10^{10}$ & 4.3003 & $1.58_{-0.28}^{+0.35}[14]$ \\
\hline
\end{tabular}

shown in Eq.1. A second effect of $\beta$ is altering the neutron to proton ratio at the freeze out temperature and this effect is limited to the electron neutrino owing to the involved weak interaction rates. The dark component will modify the expansion rate by additional effective dark fluid $\rho_{D}$ (Eq.1).The expansion rate is related to the Hubble constant $(\mathrm{H})$ via a modified Friedmann equation [1]:

$$
\left(\frac{\dot{a}}{a}\right)^{2}=H^{2}=\frac{8 \pi G}{3}\left(\rho_{\text {rad }}+\rho_{D}\right)
$$

with $\rho_{D}$ given by:

$$
\rho_{D}(T)=k_{\rho} \rho_{\text {rad }}\left(T_{0}\right)\left(\frac{T}{T_{0}}\right)^{n_{\rho}}
$$

where $T_{0}$ is chosen to be $1 \mathrm{Mev}=1.16 \times 10^{10} \mathrm{~K}, k_{\rho}$ is the ratio of effective dark fluid density over the total radiation density at $T_{0}$ and $n_{\rho}$ characterizes the adiabatic expansion of the fluid. Modifying the energy density of the universe which is radiation dominated will alter the standard-time temperature relation, $t(s)=1.39 \times \frac{k^{-0.5}}{(T(M e v))^{2}}$ [9], and consequently light element abundances. Note that $k$ is the effective number of relativistic degrees of freedom due to photon, neutrino, electron and positron degrees of freedom. In Table 2 (second and third columns) we present the effect of dark component along with neutrino chemical potentials $\beta$ (assumed to be equal for all species) where the calculated abundances agrees with observations. This is to confirm that SBBN is a rich ground for many nonstandard physics.

On the other hand, it could happen that non-equality between chemical potential still exist. For example, hypothetical neutrino-majoron coupling can reopen the window of allowing large muon/tau neutrino asymmetry together with a small electronic asymmetry ([5]). In addition, one can assume one neutrino species mixes with a sterile neutrino resulting in chaotic amplification of electron neutrino chemical potential ([15]) or assuming just a non-vanishing electron neutrino chemical potential ([16]) in a Linearly Coasting Cosmology. In a universe preferring large lepton asymmetry([6], [8]), it is important to allow neutrino chemical potential to be different in the aim to understand the SBBN 
Table 2. Effect of including non-standard neutrino properties and dark component.

\begin{tabular}{|l|l|l|l|}
\hline & \multicolumn{2}{|l|}{$n_{\rho}=6.3, k_{\rho}=0.1$} & $n_{\rho}=0, k_{\rho}=0$ \\
\hline & $N_{v}=3, \beta=0.2$ & $N_{v}=4, \beta=0.25$ & $\beta_{v_{e}}=0.23, \beta_{v_{\mu, \tau}}=2$ \\
\hline$Y_{p}$ & 0.2515 & 0.2471 & 0.2319 \\
\hline $\mathrm{D} / \mathrm{H} \times 10^{5}$ & 2.7029 & 2.9517 & 3.6530 \\
\hline${ }^{3} \mathrm{He} / \mathrm{H} \times 10^{5}$ & 1.0243 & 1.0569 & 1.1430 \\
\hline${ }^{7} \mathrm{Li} / \mathrm{H} \times 10^{10}$ & 4.3602 & 3.8121 & 2.6550 \\
\hline
\end{tabular}

and to investigate their effect on Lithium abundance. In this way we obtain constraints on these chemical potentials shown in Eq. 3 which lead to significant reduction of Lithium as shown in the fourth column of Table 2 .

$$
0.18 \leq \beta_{v_{e}} \leq 0.3, \quad 2 \leq \beta_{v_{\mu, \tau}} \leq 2.3
$$

As in most successful attempts to resolve the Lithium problem beyond the SBBN, in the present work the decrease in Lithium abundance is achieved at the expenses of increasing Deuterium. A remaining open scheme that is worth to investigate is the possibility of reducing Lithium abundance without much increase in Deuterium by including for example new particles/interactions. More detailed analysis of these non-standard physics will be presented in an upcoming paper by [18].

\section{Acknowledgement}

Tahani Makki would thank the organizing committee of the Ninth European Summer School on Experimental Nuclear Astrophysics for their support, especially she would thank prof. Rosario Gianluca Pizzone and Prof. Livio Lamia for their tremendous help.

\section{References}

[1] Arbey A. and Mahmoudi F., Phys. Lett. B 669, 46-51 (2008).

[2] Aver E., Olive K.A., and Skillman E.D., arXiv: 1503:08146 (2015).

[3] Bania T.M. et al., Nature 415, 54 (2002).

[4] Bertulani C.A. et al., arXiv:1802.03469v1, (2018).

[5] Dolgov A.D. and Takahshi F., Nucl. Phys. B, 688, 189-213 (2004).

[6] Hansen S. et al. , Phys. Rev. D 65, 023511 (2001).

[7] Hou S.Q. et al., ApJ 834, 165 (2017).

[8] Lunardini C. and Smirnov A.Yu., Phys. Rev. D 64, 073006 (2001).

[9] Mukhanov V., Physical Foundations of Cosmology (Cambridge University Press, New York, 2005) 421.

[10] Noterdaeme P. et al., A \& A 542, L33 (2012).

[11] P.A.R. Ade et al., A \& A 594, A13 (2016).

[12] Pizzone R.G., et al., ApJ 786, 112 (2014).

[13] Prantzos N. , A\&A 542, A67 (2012).

[14] Sbordone L. et al., A\&A 522, A26 (2010).

[15] Shi X., Phys. Rev. D 54, 2753 (1996).

[16] Singh P., JCAP 05, 061 (2015).

[17] Spite M. and Spite F., Nature 297, 483 (1982).

[18] T.R. Makki, M.F. El Eid, and G.J. Mathews, submitted to MNRAS (2018). 